\title{
Sweeping Jet Optimization Studies
}

\author{
LaTunia Pack Melton, Mehti Koklu†, Marlyn Andino; John C Lin§, and Louis Edelmanף \\ NASA Langley Research Center, Hampton, Virginia, United States of America
}

\begin{abstract}
Progress on experimental efforts to optimize sweeping jet actuators for active flow control (AFC) applications with large adverse pressure gradients is reported. Three sweeping jet actuator configurations, with the same orifice size but different internal geometries, were installed on the flap shoulder of an unswept, NACA 0015 semi-span wing to investigate how the output produced by a sweeping jet interacts with the separated flow and the mechanisms by which the flow separation is controlled. For this experiment, the flow separation was generated by deflecting the wing's 30\% chord trailing edge flap to produce an adverse pressure gradient. Steady and unsteady pressure data, Particle Image Velocimetry data, and force and moment data were acquired to assess the performance of the three actuator configurations. The actuator with the largest jet deflection angle, at the pressure ratios investigated, was the most efficient at controlling flow separation on the flap of the model. Oil flow visualization studies revealed that the flow field controlled by the sweeping jets was more three-dimensional than expected. The results presented also show that the actuator spacing was appropriate for the pressure ratios examined.
\end{abstract}

\section{Nomenclature}

$A_{\text {jet }} \quad$ sweeping jet orifice area, meters $^{2}$

$b$ wing span, meters

c airfoil chord (at $\delta_{f}=0^{\circ}$ ), meters

$C_{L} \quad$ lift coefficient

$C_{\mu} \quad$ SWJ actuator momentum coefficient, $\dot{m} U_{\text {jet }} /\left(S_{\text {ref }} q\right)$

$C_{p} \quad$ pressure coefficient, $\left(P-P_{s}\right) / q$

$C_{p, T E} \quad$ model trailing edge $C_{p}$

$C_{\pi} \quad$ Power coefficient, $Q * P_{s} /\left(U_{\infty} S_{\text {ref }} q\right)$

$\dot{m} \quad$ mass flow rate, $\rho U_{j e t} A_{j e t}$, grams/second

$n_{\text {jet }} \quad$ total number of sweeping jet actuators

$P \quad$ pressure, Pascals

$P_{a} \quad$ ambient pressure, Pascals

$P_{r} \quad$ pressure ratio, $P_{s} / P_{a}$

$P_{s} \quad$ actuator plenum static pressure, Pascals

$Q \quad$ Volume flow rate, meters $^{3} /$ second

$q \quad$ freestream dynamic pressure, $1 / 2 \rho U_{\infty}^{2}$, Pascals

$R e_{c} \quad$ Reynolds number based on chord

$s \quad$ wing semispan length, b/2, meters

$S_{\text {ref }} \quad$ reference surface area, meters ${ }^{2}$

$U, V \quad$ velocity components aligned with model coordinate system, meters/second

$U_{j e t} \quad$ theoretical sweeping jet actuator orifice velocity, meters/second

$U_{\infty} \quad$ average streamwise velocity, meters/second

$\alpha \quad$ angle of attack, degrees

$\delta_{f} \quad$ flap deflection angle, degrees

$\rho \quad$ density, kilograms $/$ meter $^{3}$

\footnotetext{
*Research Scientist, Flow Physics and Control Branch, Associate Fellow AIAA

${ }^{\dagger}$ Research Scientist, Flow Physics and Control Branch, Member AIAA

${ }^{\ddagger}$ Research Scientist, Flow Physics and Control Branch, Senior Member AIAA

${ }^{\S}$ Research Scientist, Flow Physics and Control Branch, Associate Fellow, AIAA

`NIFS Intern, Flow Physics and Control Branch, Student Member, AIAA
} 
$\xi / c \quad \mathrm{x}$ normalized by chord and rotated

$\psi / c \quad$ z normalized by chord and rotated

\section{A. Abbreviations}

2D two dimensional

3D three dimensional

AFC active flow control

BART Basic Aerodynamics Research Tunnel

CHL Conventional High Lift

CRM Common Research Model

FAST-MAC Fundamental Aerodynamics Subsonic/Transonic-Modular Active Control

LaRC Langley Research Center

NTF National Transonic Facility

PIV Particle Image Velocimetry

SLA stereolithography

SHL Simplified High Lift

SWJ sweeping jet

\section{Introduction}

HIGH-lift system efficiency via active flow control is being studied due to the potential benefits of impleHenting the technology on a commercial transport. ${ }^{1}$ Active flow control (AFC) for a high-lift application seeks to replace the slat and fowler flap on a conventional high-lift system (CHL) with simple hinged leading and trailing edge flaps. AFC is required for flap separation control to enable the simplified high-lift (SHL) system to generate lift comparable to the CHL system. For an AFC-enabled high-lift system to be viable, it must be both effective and efficient. In recent studies, fluidic actuators have been demonstrated to be effective at controlling separation. In this paper, we present results from on-going research to optimize and thus improve the efficiency of sweeping jet (SWJ) actuators, a type of fluidic actuator, for high-lift applications. Efficiency optimization, thus far, has focused on maximizing lift increment while minimizing SWJ power requirements.

Although fluidic actuators require an external pressure source, this technology has been used in many flow control experiments because the actuators have a simple design and can provide the control authority required. A SWJ actuator produces a jet that sweeps continuously from side to side because the actuator has no diverter at the exit. Gregory and Tomac ${ }^{2}$ provide a review of fluidic actuators that describes the history of these devices, including their current use for flow control applications. Recent AFC demonstrations that used SWJ actuators include a full-scale AFC-enhanced vertical tail project consisting of a full-scale wind tunnel test to advance the technology ${ }^{3,4,5}$ followed by a flight test. ${ }^{5}$ Fluidic actuators have also been proven to be effective in controlling flow separation thereby improving the aerodynamic performance of helicopter fuselages,${ }^{6}$ trucks,${ }^{7}$ bluff bodies,${ }^{8}$ adverse gradient ramps, ${ }^{9}$ wind turbine blades,${ }^{10}$ airfoils, ${ }^{11,12}$ and wings. ${ }^{13}$ The effectiveness of fluidic actuators is believed to be due to momentum injection into the boundary layer and the production of unsteady streamwise vorticies. These streamwise vortices interact with the separated shear layer, improving mixing between the high momentum fluid in the separated shear layer and the low momentum fluid in the boundary layer.

The SWJ actuators under investigation are similar to the curved actuator design that was used in the computational study of Vatsa et al. ${ }^{14}$ Several experimental studies have used a similar design. ${ }^{15,9,16}$ Woszidlo and Wygnanski ${ }^{11}$ used a different SWJ actuator design on an airfoil with a variable length flap chord and a variable flap deflection angle. Within the range of locations studied, the optimal excitation location was slightly downstream of the flap hinge line. They varied actuator spacing and concluded that actuator spacing was a function of jet sweep angle and the ratio of the SWJ actuator velocity to freestream velocity. Koklu ${ }^{9}$ performed a parametric study where he varied actuator size, aspect ratio, and location on a ramp model. He reported that an aspect ratio of 2 was optimal for his configuration. Varying actuator size while holding actuator spacing fixed, he showed that the AFC efficiency diminished when the actuator size was below a 
certain threshold. Seele et al. ${ }^{17}$ varied actuator size and spacing on a swept wing configuration and found that their larger actuators were more efficient at controlling separation. A recent study of Hartwich et al. ${ }^{18}$ suggests that additional research may be needed to optimize actuator geometry and spacing for high-lift applications. The CFD study shows that while SWJ actuators can be used to obtain the lift that would be required to replace a conventional high-lift system with an AFC-enabled high-lift system, the required mass flow exceeded the amount available on an aircraft by an order of magnitude. In this study, we vary actuator geometry for a fixed actuator orifice size to determine how the sweep angle and jet momentum produced by the different actuator geometries influence the separated flow field being controlled.

The SWJ actuators being investigated have an orifice width of $2 \mathrm{~mm}$ and height of $1 \mathrm{~mm}$. The orifice size corresponds to that which will be used on the outboard region of the Fundamental Aerodynamics Subsonic/Transonic-Modular Active Control (FAST-MAC) model ${ }^{19,20}$ for an upcoming high Reynolds number AFC wind tunnel experiment in the National Transonic Facility (NTF). Recent studies including that of Melton, ${ }^{13}$ Koklu, ${ }^{9}$ and Melton and Koklu ${ }^{16}$ have noted that as actuator scale decreases, the sweep angle of the actuator decreases. The impact of this change is a potential reduction in the efficiency of the AFC system resulting from suboptimal actuator spacing. The original plan for the NTF AFC experiment was to use the curved SWJ design used in previous studies. However, the small sweep angle observed during benchtop testing of the actuator led us to explore other geometries for the outboard region of the FASTMAC model. In this paper, we compare benchtop data and wind tunnel data from the candidate actuator geometries investigated.

\section{Experiment Description}

\section{A. Wind Tunnel Description}

The experiments were conducted in the NASA Langley Research Center (LaRC) Basic Aerodynamics Research Tunnel (BART). The open-circuit tunnel has an 11:1 contraction ratio and a test section that is 0.71 $\mathrm{m}$ high by $1.02 \mathrm{~m}$ wide by $3.05 \mathrm{~m}$ long. BART is used primarily as a flow physics facility; therefore, it has the instrumentation and optical access needed for measurement techniques such as Laser Doppler Velocimetry (LDV) and Particle Image Velocimetry (PIV). The maximum velocity of the tunnel is approximately $60 \mathrm{~m} / \mathrm{s}$ and we tested at speeds up to $50 \mathrm{~m} / \mathrm{s}$.

\section{B. Model Description}

The model tested is a $0.305 \mathrm{~m}$ chord (c), $0.610 \mathrm{~m}$ semipan (s) wing with a NACA 0015 cross-section that was built for sidewall-mounted AFC testing in the BART facility. ${ }^{21,22,23}$ The wing is unswept and has a $30 \%$ chord trailing edge flap that can be tested at flap deflection angles, $\delta_{f}$, from $-10^{\circ}$ to $40^{\circ}$ in $10^{\circ}$ increments. The $0.61 \mathrm{~m}$ span flap is split into three equal-span components that can be deflected independently. The model has four rows of streamwise pressure taps at spanwise $(y / s)$ locations of -0.17 , $0.50,-0.83$, and -0.99 and four rows of spanwise pressure taps at streamwise $(x / c)$ locations of 0.0050 , $0.30,0.77$, and 1.0. A 5-component strain gauge balance was used to measure forces and moment on the model.

During the SWJ actuator study using the swept wing version of the model, ${ }^{13}$ the existing aft region of the model was modified so that SWJ actuators

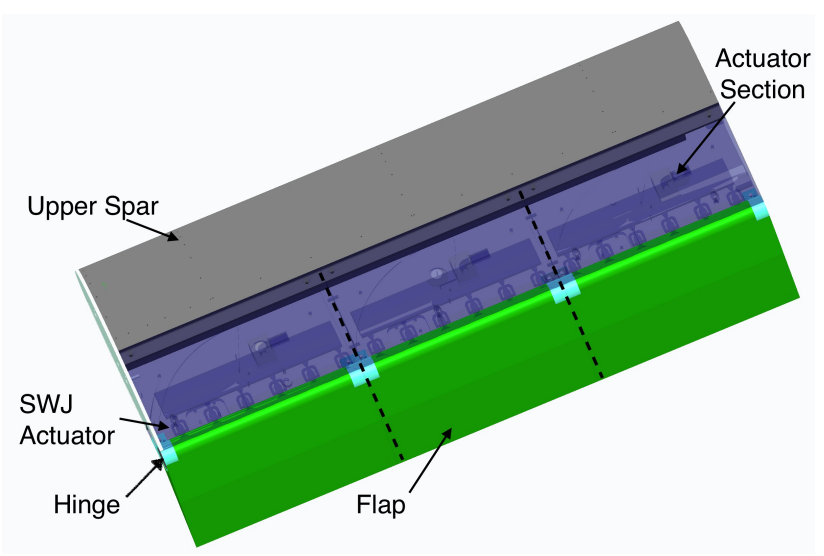

Figure 1. CAD image of NACA 0015 semispan wing model with dashed lines indicating SWJ actuator sections, flap sections and hinge locations. could be placed in the aft region of the main element of the model, along the flap shoulder. However, due to the small size of this region, actuator options were limited. For the current set of experiments, the upper spar, manufactured to enable the aft section of the main spar to be removed and replaced with various actuator options, was used. ${ }^{16}$ The new actuator modules were made using high-resolution stereolithography (SLA). A CAD rendering of the model is shown in Fig. 1. 


\section{SWJ Actuators}

The SLA actuator modules were built in three sections and are located upstream of the flap shoulder as shown in Fig. 1. Each section was $0.20 \mathrm{~m}$ wide and covered the span of a flap section. The SWJ actuators selected for this study are shown in Fig. 2. The original actuator (Fig. 2(a)), a scaled version of the one used in the studies of Koklu and Melton ${ }^{24}$ and Vatsa et al. ${ }^{14}$ has a smaller sweep angle as the jet does not attach to the internal Coanda surface. The jet oscillation and small sweep angle are generated only by the deflection of the main jet at the second throat due to backflow in the feedback loops. The internal Coanda surfaces and external diffuser walls of the modified actuators are adjusted to take advantage of the Coanda effect enabling the deflected jet to attach to the internal Coanda surfaces and achieve a larger sweep angle. The actuator studied in Ref. 24 had an aspect ratio of 1, meaning the orifice width was equal to the orifice height. In this study, we used actuators that had orifice width to height ratios of 2:1. This ratio was chosen because Koklu and Owens ${ }^{25}$ found it was the most effective at controlling separation on their ramp model. The Mod 1 actuator depicted in Fig. 2(b) is a variation of the original actuator where the spacing between the two internal Coanda surfaces is reduced. The Mod 2 actuator geometry (Fig. 2(c)) is a different actuator geometry that has been used by the auto industry. The shape and spacing of the internal coanda surfaces differs from that of the original geometry. Table 1 summarizes the differences in the actuator geometries. Six actuators were in each SLA flap section. The spacing between each actuator was $3.3 \mathrm{~cm}$ which is similar to what will be used during the high Reynolds number (FAST-MAC) AFC wind tunnel experiment.

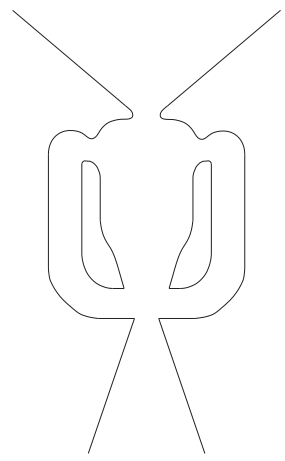

(a) Original

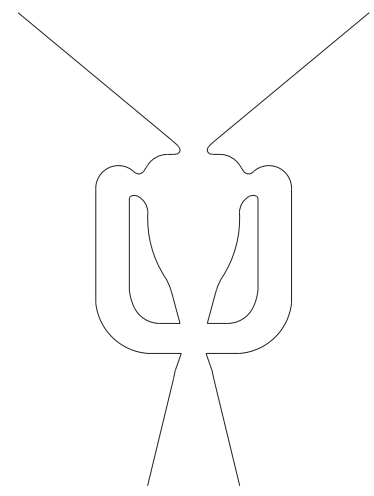

(b) Mod 1

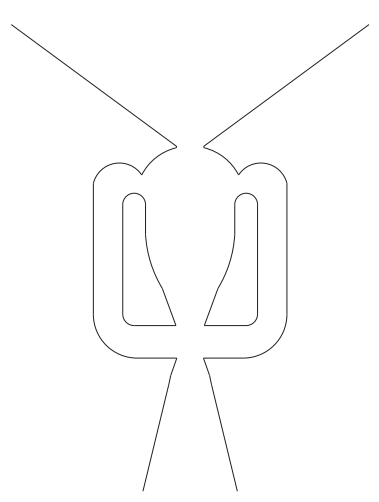

(c) Mod 2 .

Figure 2. Actuator geometries.

Table 1. SWJ actuator dimensions.

\begin{tabular}{|c|r|r|r|r|}
\hline SWJ Actuator & Geometry & $\theta_{\text {diff }}$ & $w_{c}, \mathrm{~mm}$ & $h_{f t}, \mathrm{~mm}$ \\
\hline & & & & \\
\hline
\end{tabular}

Each of the three actuators sections was independently controlled by an electronic pressure regulator. Thermal mass flow meters in the three supply lines were used to measure flow rate and each actuator plenum was instrumented with a static pressure orifice and a thermocouple that were used to compute density. The jet velocity is defined by Eq. 1 , where, $A_{\text {jet }}$ is the actuator orifice area, $2 \mathrm{~mm}^{2}$, and density is computed using isentropic relations. $C_{\mu}$ is defined by Eq. 2. Several recent SWJ actuator AFC studies ${ }^{3,4}$ have used 
similar definitions for $C_{\mu}$. Variations of this definition have also been used where density, $\rho$ is assumed to be constant.

$$
\begin{gathered}
U_{j e t}=\frac{\dot{m}}{\rho n_{j e t} A_{j e t}} \\
C_{\mu}=\frac{\dot{m} U_{j e t}}{q S_{r e f}}
\end{gathered}
$$

The actuator jet exited the model at the flap shoulder and was nearly tangential to the flap surface when the flap deflection angle, $\delta_{f}$, was $0^{\circ}$. The actuators were integrated into SLA parts so that the outer mold line of the model did not change. Integrating actuators with a height larger than the original slot height of $0.76 \mathrm{~mm}$ into the model required that the SWJ actuator slot heights converge from $1.0 \mathrm{~mm}$ to $0.50 \mathrm{~mm}$ between the nozzle orifice and the actuator exit. The new slot height of $0.5 \mathrm{~mm}$ is smaller than the original slot height because, for durability reasons, the SLA parts were manufactured with trailing edge thicknesses larger than those used on the original stainless steel part.

\section{SWJ Actuator Characterization Studies}

Hotwire surveys were performed at $P_{r}=1.07$ to narrow the list of candidate actuators to the three that are the focus of this paper. From these surveys, the jet sweep angles of the three actuators were determined to be $60^{\circ}, 106^{\circ}$ and $110^{\circ}$ for the original, Mod 1, and Mod 2 geometries, respectively. A custom-made total pressure probe with a flattened hypordermic tube tip based on the design of Owens et al. ${ }^{26}$ was used to measure the total pressure at the actuator exit. Total pressure surveys were performed because the hotwire probes were too fragile to be used over the pressure range investigated. In addition to the custom total pressure probe having the spatial resolution needed to survey the $1 \mathrm{~mm} \times 2 \mathrm{~mm}$ actuators studied, the probe does not exceed the slot height of the actuator. The probe design incorporates an unsteady pressure transducer that is used to measure the frequency of the SWJ actuators. Since the probe was not dynamically calibrated, it cannot be used to measure time dependent total pressure fluctuations. Figure 3 presents SWJ actuator frequency as a function of $P_{r}$, for a single actuator in the inboard actuator section that was used in the wind tunnel. There are two curves for each actuator geometry with one curve representing data acquired from benchtop surveys and the other curve representing data acquired from the unsteady pressure transducer located at the flap shoulder near the center span of the inboard flap. Similar results from benchtop testing indicate that all three actuators oscillate at close to the same frequency. The original actuators that were used for wind tunnel testing oscillated at higher frequencies as shown in Fig. 3. The reason for the difference in frequencies is still being investigated.

Total pressure surveys were performed in a zigzag pattern to minimize travel distance along a trapezoidal-shaped grid in the xy plane. The $\mathrm{x}$ resolution was initialized at $\Delta x=0.25 \mathrm{~mm}$ and increased by $10 \%$ at each subsequent grid line from $\mathrm{x}=0.25 \mathrm{~mm}$ to $20 \mathrm{~mm}$. The $\mathrm{y}$-resolution was initialized at $\Delta y=0.25 \mathrm{~mm}$ along a defined grid base width. Each subsequent line of the grid was widened by an expansion angle $\theta=20^{\circ}$ maintaining a constant number of y-grid points in each line. The results from these mean pressure surveys are presented in the contour plots of Fig. 4. Pressure contours for two pressure ratios are presented to highlight the differences in the averaged jet produced by the three actuators as well as the changes in the shape of the mean jet with increasing supply pressure. The jet deflection or sweep angle of the Mod 2 actuator is larger than that of the other two actuators. Melton and Koklu ${ }^{16}$ examined the effect of jet deflection an-

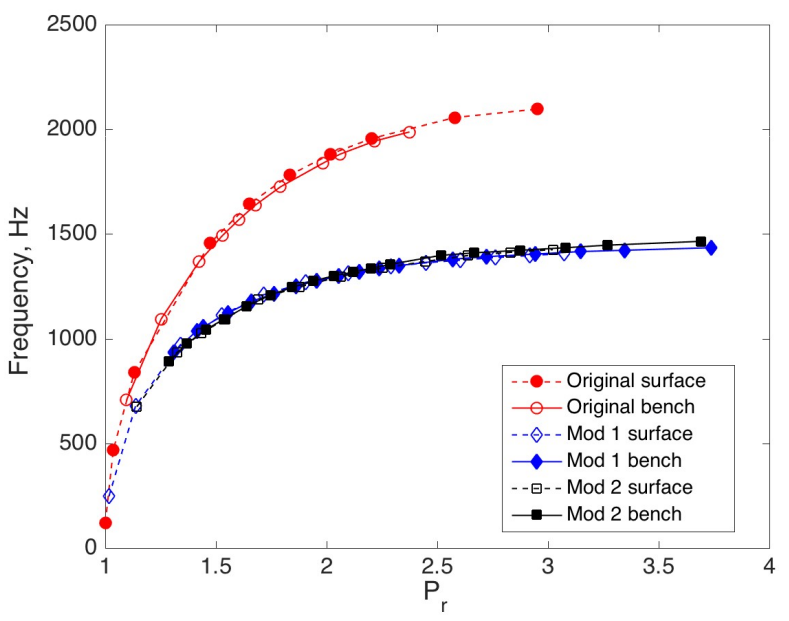

Figure 3. Actuator frequency as a function of $P_{r}$ for the three actuator geometries. 


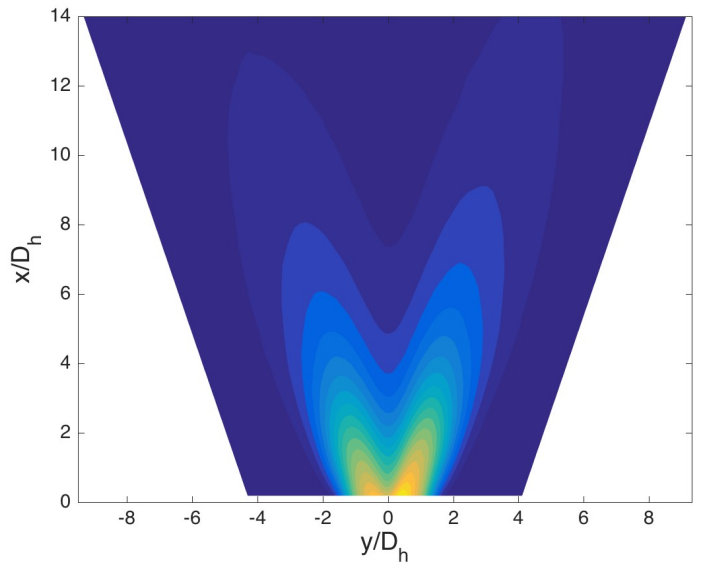

(a) Original, $P_{r}=1.5$.

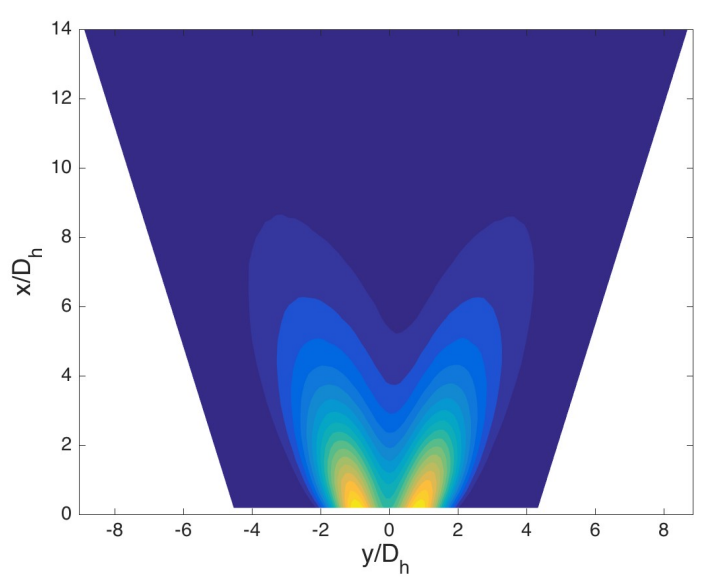

(c) Mod 1, $P_{r}=1.5$.

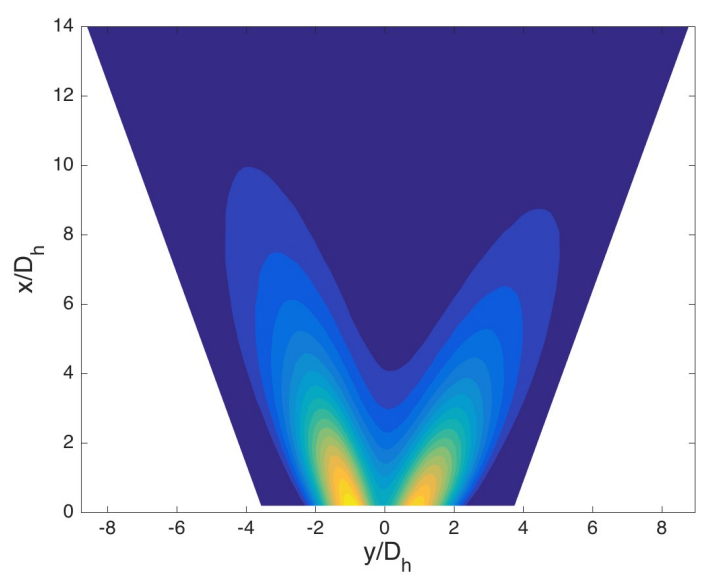

(e) $\operatorname{Mod} 2, P_{r}=1.5$.

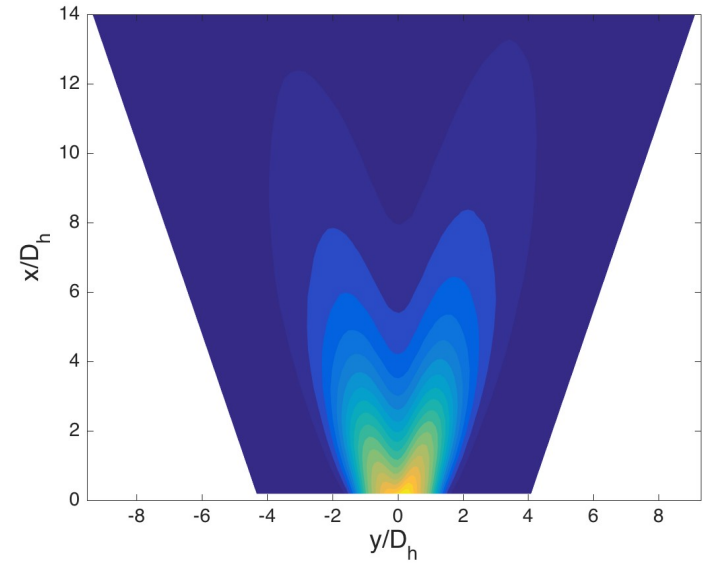

(b) Original, $P_{r}=1.8$.

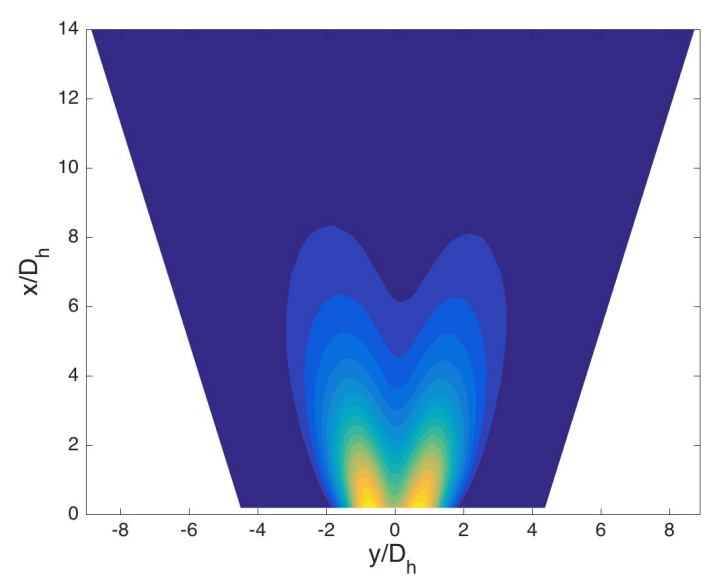

(d) $\operatorname{Mod} 1, P_{r}=1.8$.

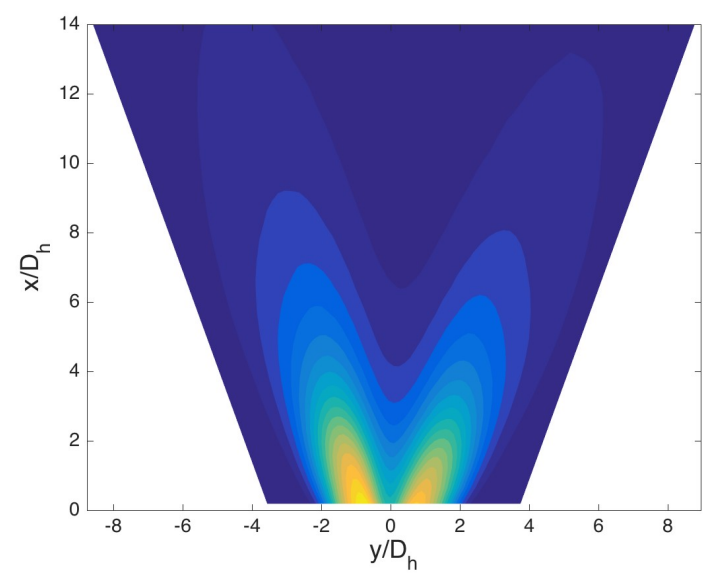

(f) $\operatorname{Mod} 2, P_{r}=1.8$.

Figure 4. Total pressure contours of the three SWJ actuator geometries. 
In this set of experiments deflection angle was varied while fixing the orifice size of the actuator geometry. After documenting the performance of each of the actuators, the next step was to determine what impact, if any, the variations in jet flowfields and jet deflection angles had on the efficiency of the SWJ actuator AFC system.

\section{Flow Control Results}

To compare the AFC performance of the three actuator geometries, the flap deflection, $\delta_{f}$, was set at $40^{\circ}$, the maximum flap deflection of the model. At $\delta_{f}=40^{\circ}$, the flow is separated over most of the flap chord. This flap deflection was selected because of the current interest in assessing the performance of the SWJ actuators on models with large flap deflections and hence large adverse pressure gradients. This interest stems from a desire to augment the existing AFC dataset obtained on the FAST-MAC model using steady blowing at flap deflection angles of $30^{\circ}$ and $60^{\circ}$ with SWJ AFC data. Another reason for our interest in large flap deflections is that Hartwich et al. ${ }^{18}$ used a Common Research Model (CRM) based high-lift configuration with a trailing edge flap deflection of $50^{\circ}$ for their computational study on a SHL configuration. Design is underway for a $10 \%$ scale semispan wing wind tunnel model for this CRM-based high-lift configuration. Therefore, experimental and computational studies are needed to develop efficient and effective AFC actuation systems for the model.

Melton and Koklu ${ }^{16}$ used the original actuator geometry to control separation on the trailing edge flap and showed that the actuators were effective at controlling separation at pre-stall angles of attack $\left(-4^{\circ} \leq \alpha \leq 12^{\circ}\right)$ over the entire freestream velocity range investigated $\left(U_{\infty} \leq 35 \mathrm{~m} / \mathrm{s}\right)$. Thus, the efficiency of the SWJ actuators was primarily investigated with the model at $\alpha=8^{\circ}$ and $R e_{c}=500,000\left(U_{\infty}=25 \mathrm{~m} / \mathrm{s}\right)$. Due to the range limitations of the balance and pressure instrumentation, the angle of attack of the model was reduced to $\alpha=4^{\circ}$ to compare actuator AFC performance at elevated Mach numbers.

\section{A. Actuator Efficiency}

Figure 5(a) shows mean contours of streamwise velocity from PIV data over the flap of the model at $y / s=-$ 0.48 and illustrates the large separated region on the flap at these conditions. The PIV data show that flow separation occurs downstream of the flap hinge line. Since the flow separates downstream of the flap shoulder, placing the SWJ actuators closer to the separation location should improve the efficiency of the AFC system. Actuation from the flap shoulder was chosen for the current study because of the ease with which we could vary actuator geometries. The SWJ actuator sections shown in Fig. 1 can be installed without removing the model from the tunnel. Additionally, the flap size limits actuator size and the flap rotation limits the air supply to a single input location, thereby eliminating the flexibility of varying the SWJ output at multiple locations along the span of the model. The baseline pressure distributions at $y / s=-0.5$ are presented in Fig. 5(b) and also indicate that the flow is separated on the flap. Mean streamwise velocity contours are presented in Fig. 5(c) for the controlled flowfield when the trailing edge pressure coefficient at $y / s=-0.5$ is 0 (see Fig. 5(d) for the corresponding pressure distribution). The Mod 2 actuator is used to control the flow. The PIV results indicate that SWJ actuators eliminated the separated flow on the flap, and the pressure distribution indicates that AFC generates a pressure recovery on the flap of the model.

Figures 6(a) and 6(b) present the lift increment obtained on the NACA 0015 semispan wing model using the three actuator configurations shown in Fig. 2 when the mass flow rate and thus momentum coefficient, $C_{\mu}$, were varied. The data show that the original actuator requires more momentum and more mass flow than the two modified actuators. The data suggest that with the fixed spacing of $3.3 \mathrm{~cm}$, the modified actuator geometries are more efficient, in terms of mass flow and momentum, at controlling separation on the model than the original actuator geometry. The power coefficient, $C_{\pi}$, is another parameter used to compare the efficiency AFC actuation systems. For the sweeping jet actuator, $C_{\pi}$ is a function of both actuator plenum pressure and volume flow rate. This is beneficial, especially when comparing SWJ actuators with different size orifices. Actuators with different sizes were compared in Reference 16 and the smaller actuators required less mass flow and momentum but consumed more power than the larger actuators due to the higher plenum pressure requirements of the smaller actuators. Fig. 6(c) indicates that the Mod 1 and Mod 2 actuators require less power to increase $C_{L}$ than the original actuator. Since the actuators have the same orifice size, the plenum pressure requirements are similar and thus the mass flows, momentum coefficients, and power coefficients have the same trends. 




(a) Baseline PIV.

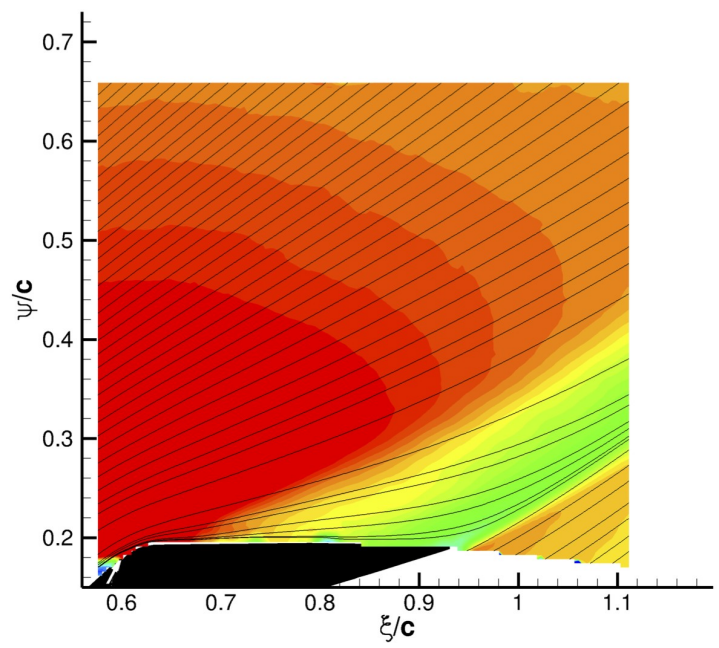

(c) Control PIV.

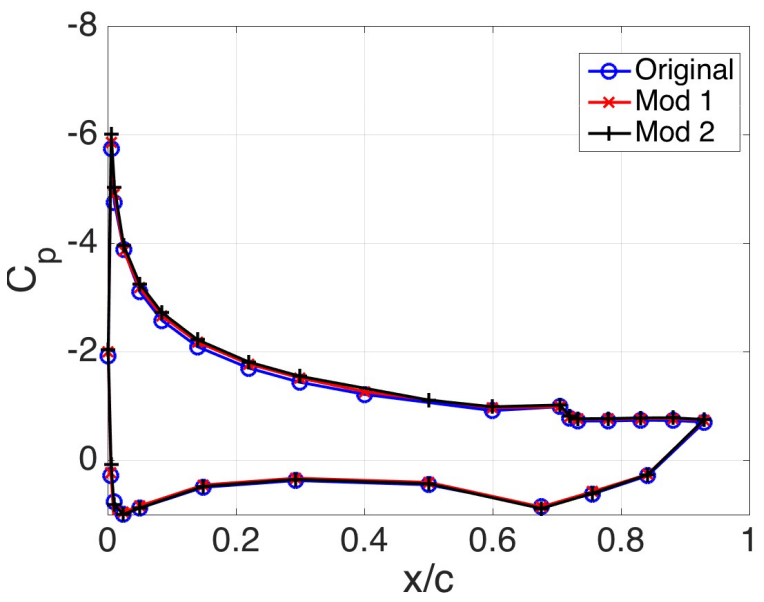

(b) Baseline $C_{p}$.

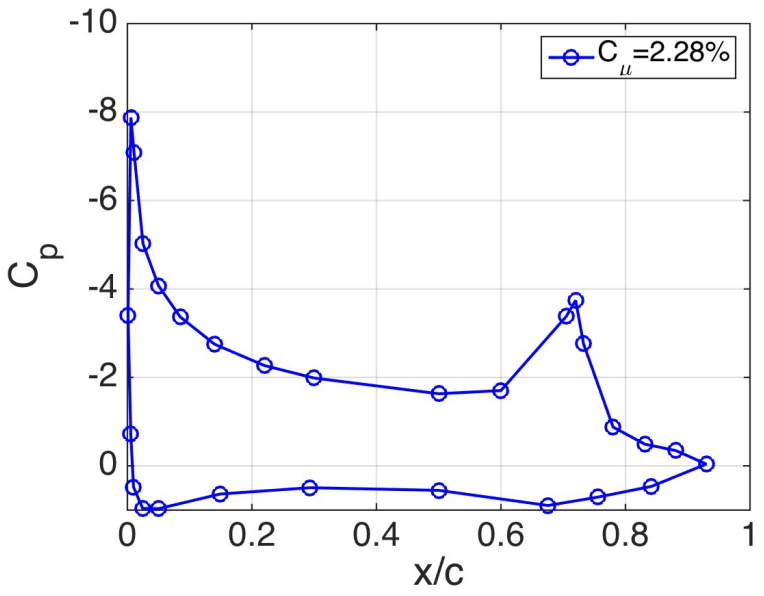

(d) Control $C_{p}$.

Figure 5. Mean streamwise velocity contours on the flap from PIV measurements and pressure distributions for the baseline and AFC cases. $R e_{c}=\mathbf{5 0 0 , 0 0 0 ,} \delta_{f}=40^{\circ}, \alpha=8^{\circ}, y / s=\mathbf{- 0 . 4 8}$ for PIV and $y / s=-\mathbf{0 . 5 0}$ for $C_{p}$. 
Figure 7 presents $C_{p}$ distributions for selected $C_{\mu}$ levels for the three actuators. The $C_{p}$ distributions are from the four rows of streamwise pressure orifices along the model span. The $C_{\mu}$ levels presented range from 0 (baseline case) to the level required to achieve a trailing edge $C_{p}, C_{p, T E}$, of zero at the three most inboard locations $(y / s=-0.33,-0.50$, and -0.83$)$. The maximum $C_{\mu}$ levels presented are not the maximum levels that the AFC system was capable of producing. The lower suction peak at the leading edge and along the suction surface of the model for the two most outboard streamwise pressure locations show that less lift is generated on the outboard portion of the wing with and without AFC. The most efficient actuator, Mod 2, produces the largest suction peak at the flap shoulder for all of the AFC cases presented. The large suction peaks at the flap shoulder produced by the Mod 1 and Mod 2 actuators are indicators of the effectiveness of the actuation. Since the mass flow rate to each flap section could be independently controlled, once $C_{p, T E}=0$, the input level at that location was not increased. For the Mod 1 and Mod 2 actuators the ability to individually control each section of the flap is evidenced by identical $C_{p}$ distributions for the highest two $C_{\mu}$ level presented at $y / s=-0.83$ and $y / s=-0.99$. In these cases the inboard portion of the flap required higher levels of $C_{\mu}$ to achieve $C_{p, T E}=0$.

The angle of attack of the model was reduced to $\alpha=4^{\circ}$ to compare the efficiency of the three actuators at higher Mach numbers. Figures 8(a), 8(c), and 8(b) show the lift increment obtained using the actuators with the model angle of attack, $\alpha$, at $4^{\circ}$. The data confirm that the actuators are effective over a range of angles of attack. Since the AFC results indicate that the Mod 2 geometry performed slightly better, we selected this geometry for the upcoming high Reynolds number AFC experiment. Therefore, the remainder of the paper will focus on differences between the Mod 2 configuration and the original actuator configuration.

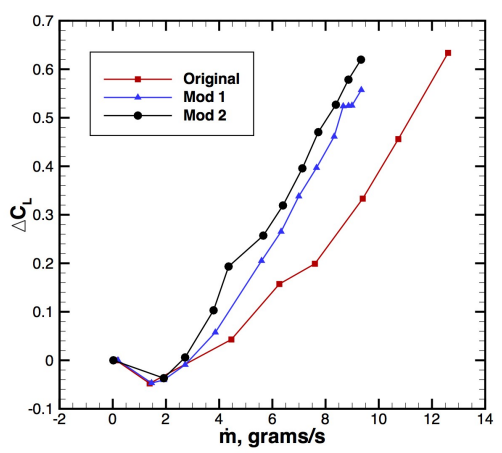

(a) Mass Flow Rate.

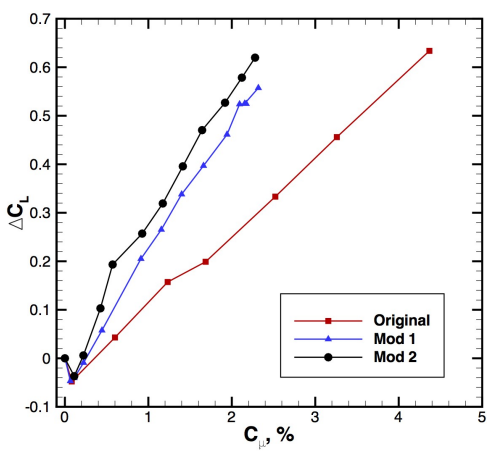

(b) Momentum Coefficient.

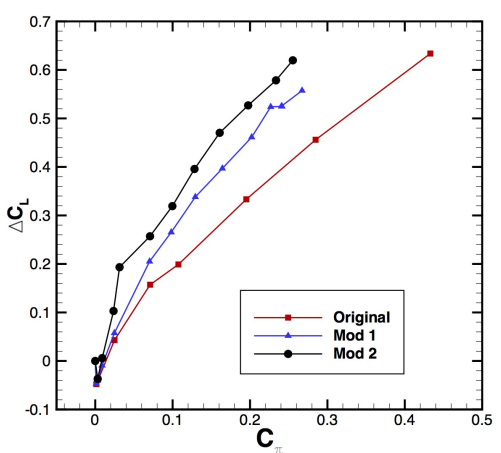

(c) Power Coefficient.

Figure 6. Lift increment of the three actuator geometries. $R e_{c}=500,000, \delta_{f}=40^{\circ}$, and $\alpha=8^{\circ}$.

\section{B. Flow Visualization}

To explore the theory that actuator spacing was not optimized for the original actuator, we performed oil flow visualization studies to compare the performance of the Mod 2 actuator and the original actuator. We applied the mixture of oil and fluorescent pigment to the region downstream of the actuator nozzle to reduce the risk of contaminating the pressure and actuator orifices. Kapton tape covered the pressure orifices on the model. Fig. 9(a) and Fig. 9(b) present the flow field produced by the original and Mod 2 actuators in the absence of a freestream velocity. The flow is from top to bottom. Note that the dark region near the center span of the flap is where the surface was painted black for PIV. The images show that the spacing between actuators for these pressure ratios provides complete coverage between actuators, thus eliminating actuator spacing as the sole reason for the difference in performance of the two actuators. The region of influence of each SWJ actuator is easily detected, because the line between each actuator is marked by dark lines in the flow visualization images. Fig. 9(a) shows that one of the SWJ actuators in the inboard region of the model did not sweep. This was not due to SWJ actuator failure. Repeat flow visualization runs revealed that the SWJ motion could be influenced by the steps produced by the flap hinges that divide the flap into three sections and the tape in the streamwise direction used to cover the pressure orifices. 


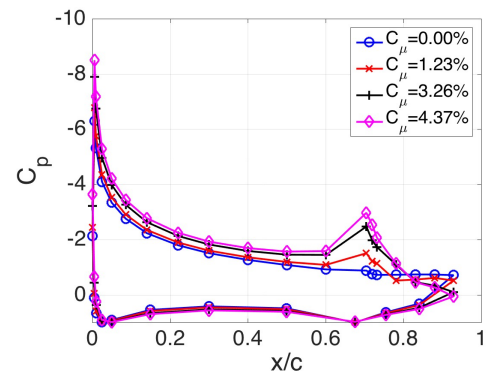

(a) $y / s=\mathbf{- 0 . 3 3}$, Original.

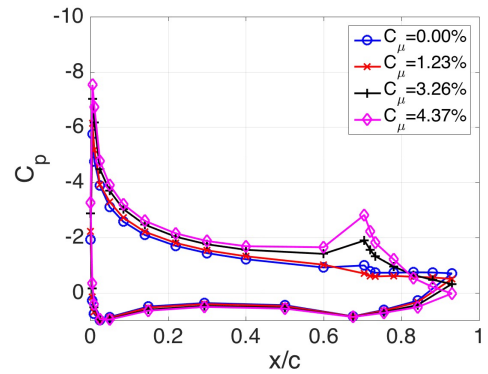

(d) $y / s=-\mathbf{0 . 5}$, Original.

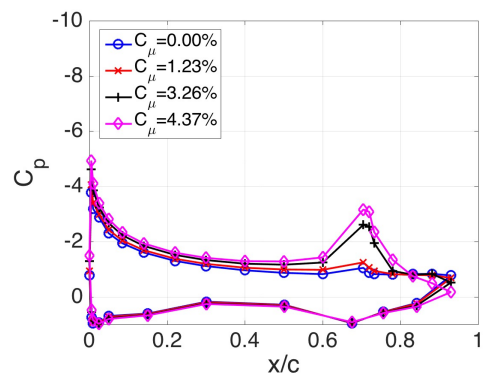

(g) $y / s=\mathbf{- 0 . 8 3}$, Original.

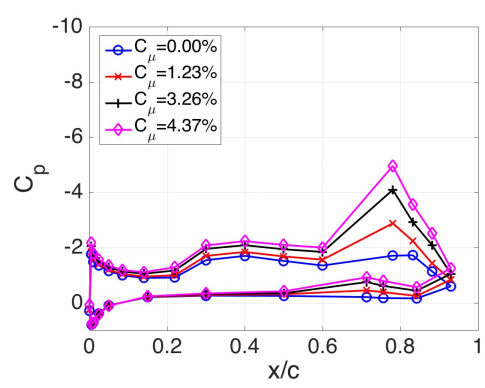

(j) $y / s=-\mathbf{0 . 9 9}$, Original.

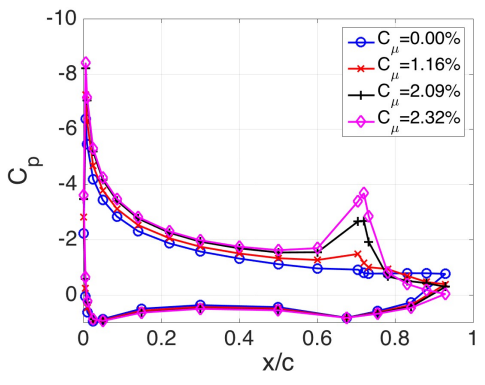

(b) $y / s=-\mathbf{0 . 3 3}$, Mod 1 .

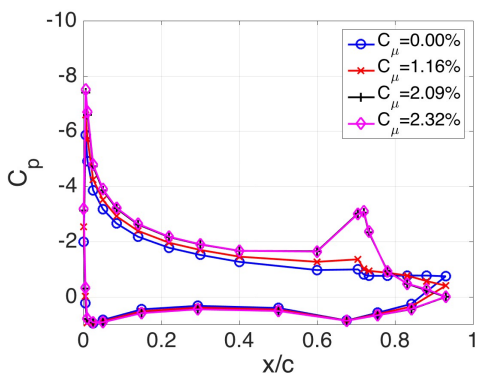

(e) $y / s=-\mathbf{0 . 5}$, Mod 1 .

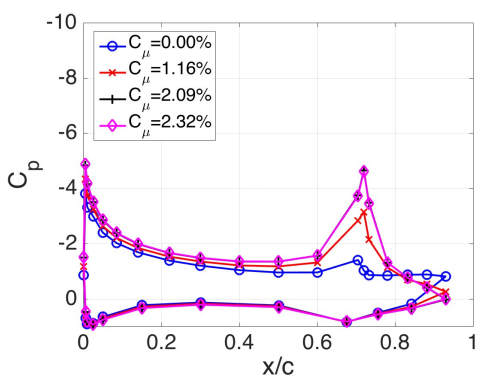

(h) $y / s=\mathbf{- 0 . 8 3}$, Mod 1.

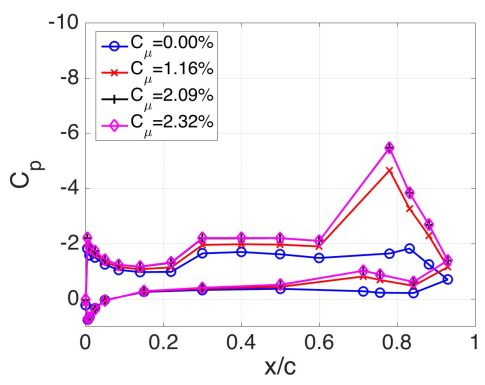

(k) $y / s=-\mathbf{0 . 9 9}$, Mod 1 .

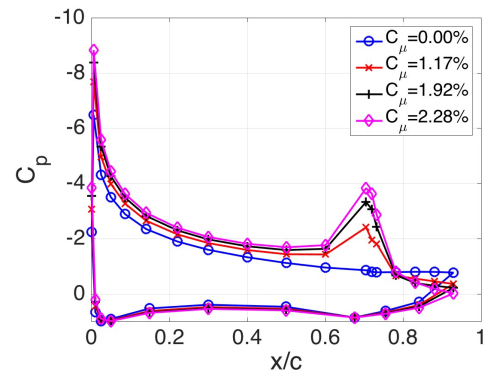

(c) $y / s=-\mathbf{0 . 3 3}$, Mod 2.

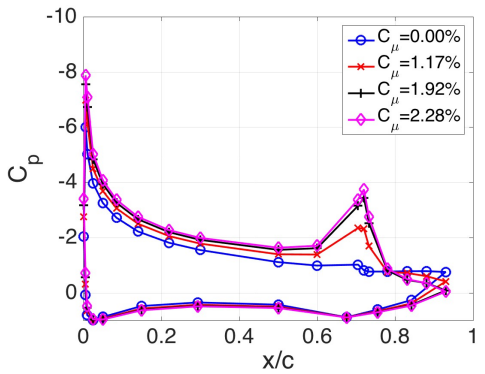

(f) $y / s=-0.5$, Mod 2.

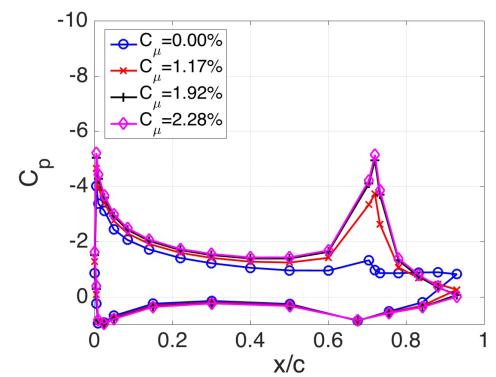

(i) $y / s=-\mathbf{0 . 8 3}$, Mod 2 .

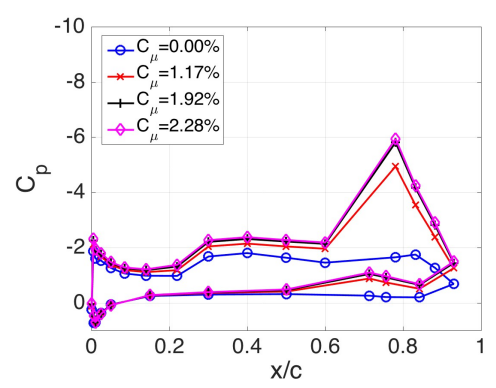

(l) $y / s=-\mathbf{0 . 9 9}$, Mod 2.

Figure 7. Wing pressure distribution for selected momentum coefficient levels. $R e_{c}=\mathbf{5 0 0 , 0 0 0 ,} \delta_{f}=40^{\circ}$, and $\alpha=8^{\circ}$. 


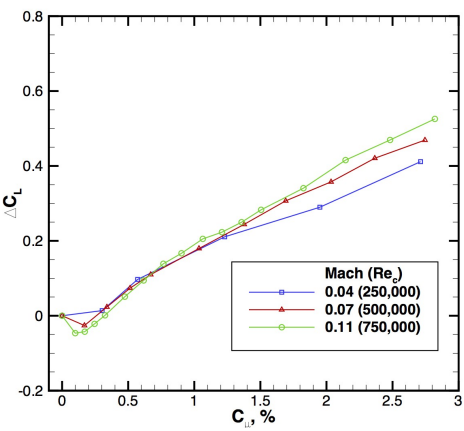

(a) Original.

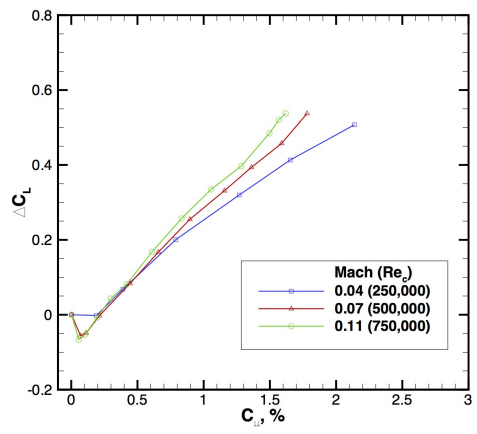

(b) Mod 1.

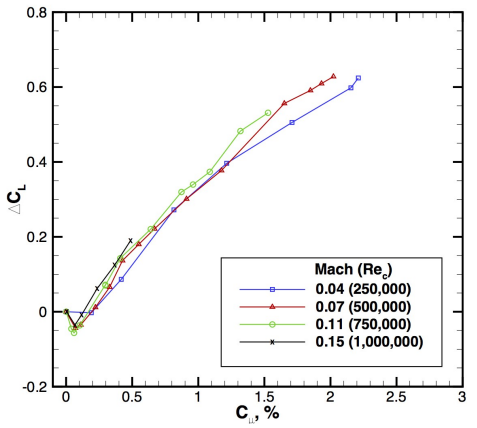

(c) Mod 2.

Figure 8. Lift increment for the modified actuators with Mach number varied. $\delta_{f}=40^{\circ}$, and $\alpha=4^{\circ}$.

Figure 9(c) and 9(d) provide a overall view of how the SWJ actuators attach the flow on the flap. Attached flow is seen over a large portion of the flap span at the pressure ratios presented. Near the flap shoulder, at the boundary between SWJ actuators necklace vortices are present. Similar vortices were observed by Woszidlo and Wygnanski. ${ }^{11}$ Koklu $^{9}$ also observed similar vortices when using micro vortex generators. The vortices usually appear at the separation locations. There are also local separation regions near the trailing edge of the flap that appear to originate from either the flap junctions or the tape edges. In these two cases, unlike the $\mathrm{M}=0.0$ oil flow results of Fig. 9 (a), there is no indication that the jets are not sweeping. The trailing edge pressure distributions are presented in Fig. 10 and confirm that there are local regions of separation at the flap trailing edge that appear to originate from steps around the flap hinges. More recent results from experiments using a single $0.61 \mathrm{~m}$ span flap and the Mod 2 actuator reveal that the vortices are present when there are no hinges along the flap span. Therefore, the vortices may originate from the SWJ actuators. If the vortices were distributed along the model span the results would be similar to the vortices produced when using vortex generators that are too large. A region of low pressure near the tip of the model due to the tip vortex is shown in the baseline data of Fig. 10. SWJ actuation appears to change the location of the tip vortex shown in the baseline $C_{p}$ data. When control is applied, a corner vortex near the inboard region due to the wing-endplate juncture is depicted by the low pressure region at $y / s=-0.083$.

Two dimensional (2D) PIV was also used to aid in visualizing the vortices produced by the actuator. Figs. 11(a) and 11(b) present mean vorticity contours for the original and Mod 2 actuators. The PIV setup described in Ref. 16 was used. The camera field of view is roughly $50 \mathrm{~mm}$ x $50 \mathrm{~mm}$ and was used to focus on one SWJ actuator along the span of the model. The results, acquired at the same streamwise location, show that the two geometries produce jets that interact with the flowfield in a very different manner. Control using the Mod 2 actuator (Fig. 11(b)) produces a pair of counter rotating vortices, near the centerline, that appear to be very similar suggesting that their strength is comparable. The pair of vortices at $y / s=-0.43$ are not as distinct. The vortices are similar to those shown in Ref. 16 for the original geometry actuator with an orifice size of $2 \mathrm{~mm} \times 4 \mathrm{~mm}$. The original actuator produces additional vortices between the actuators that require more investigation at multiple locations along the flap chord.

\section{Conclusions}

As part of a larger experimental test campaign to determine the appropriate SWJ actuators to use in an upcoming high Reynolds number AFC wind tunnel experiment, we studied the performance of three SWJ actuator geometries. The actuator geometries, with the same orifice size $(2 \mathrm{~mm}$ wide $\mathrm{x} 1 \mathrm{~mm}$ high), were investigated to determine how the jet produced by the actuator influenced the separated flowfield on the deflected flap of an unswept, semispan wing. Benchtop experiments were performed on the original candidate actuator and showed that the actuator produced an oscillating jet that had a smaller sweep angle than expected. Two additional actuators were selected for this study as they produced jets with larger sweep angles at low pressures. This difference in jet sweep angle for actuators with the same orifice size provided an opportunity to study the influence of sweep angle on AFC efficiency. The wind tunnel experiments showed that both modified actuators required less mass flow and momentum to control the separated flow on the 




(a) Original actuator, $P_{r}=\mathbf{1 . 6}, \mathbf{M}=\mathbf{0}$.

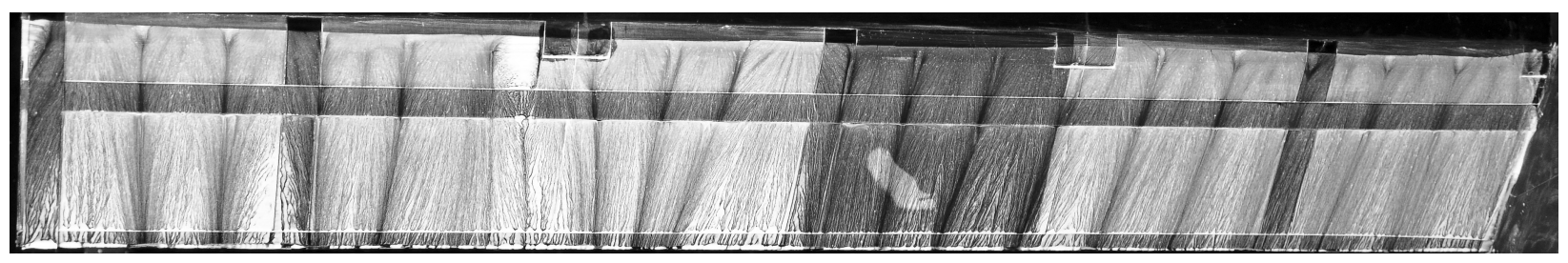

(b) Mod 2 actuator, $P_{r}=1.7, \mathrm{M}=\mathbf{0}$.

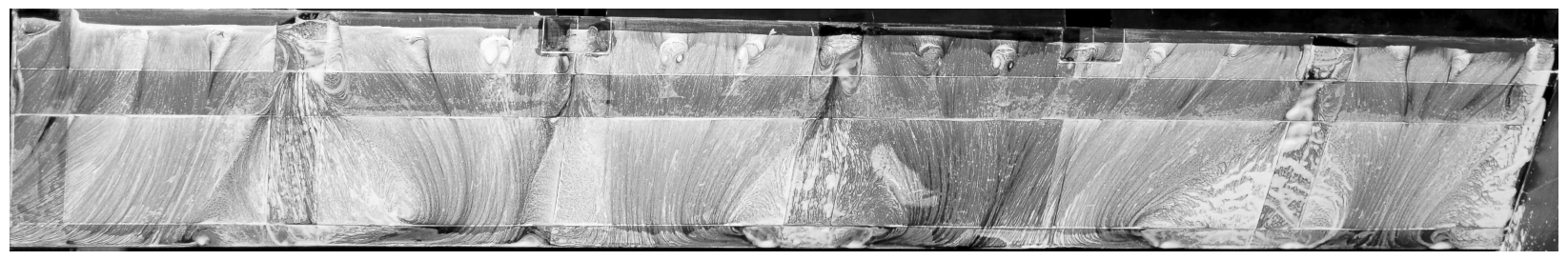

(c) Original actuator, $P_{r}=1.6, \mathrm{M}=\mathbf{0 . 0 7}$.

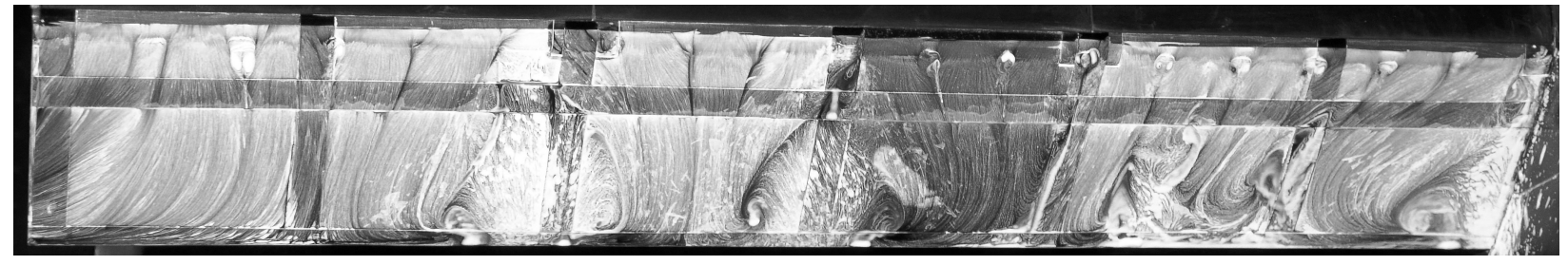

(d) Mod 2 actuator, $P_{r}=1.7, \mathrm{M}=0.07$.

Figure 9. Surface oil flow visualization.

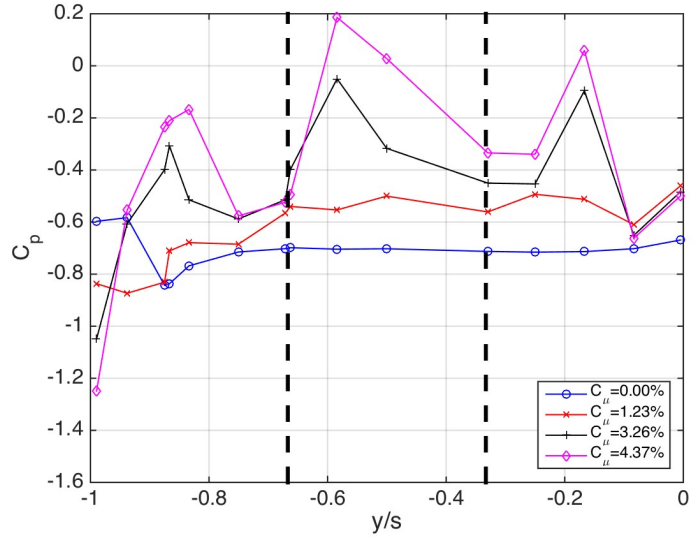

(a) Original Actuator.

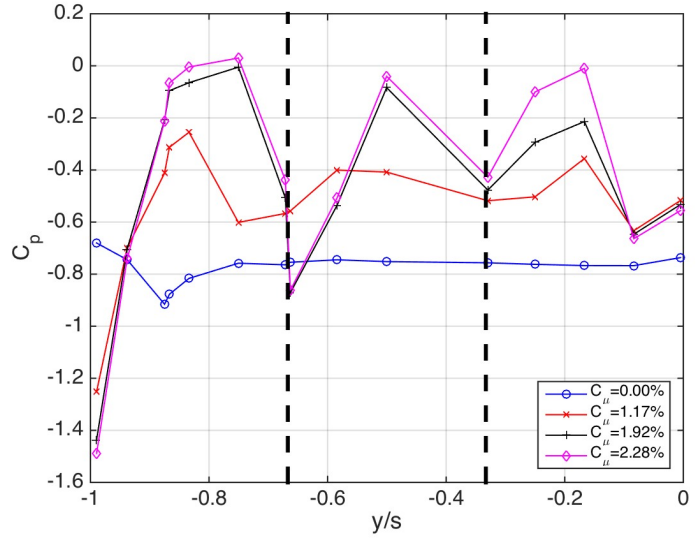

(b) Mod 2 Actuator.

Figure 10. Trailing edge spanwise pressure distributions with SWJ control. $R e_{c}=500,000, \mathrm{M}=0.07$. Vertical dashed lines represent flap hinge locations. 


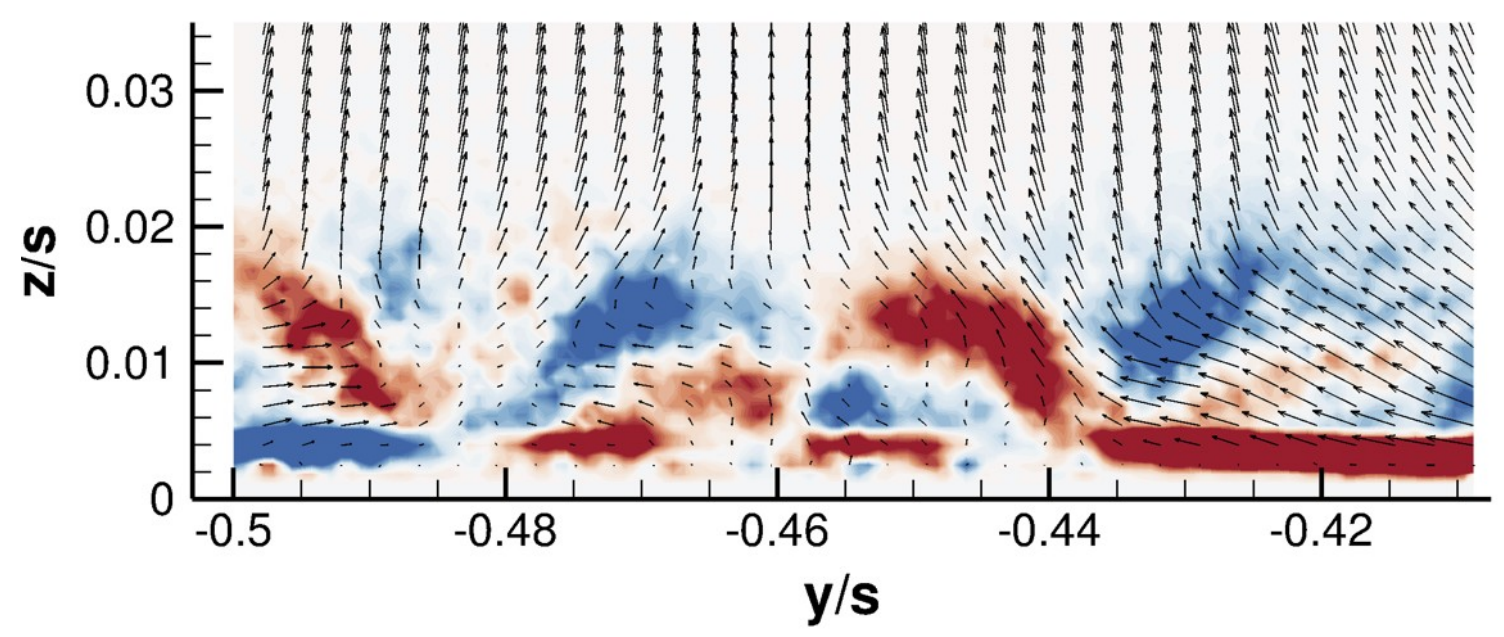

(a) Original Actuator, $P_{r}=\mathbf{1 . 8}$.

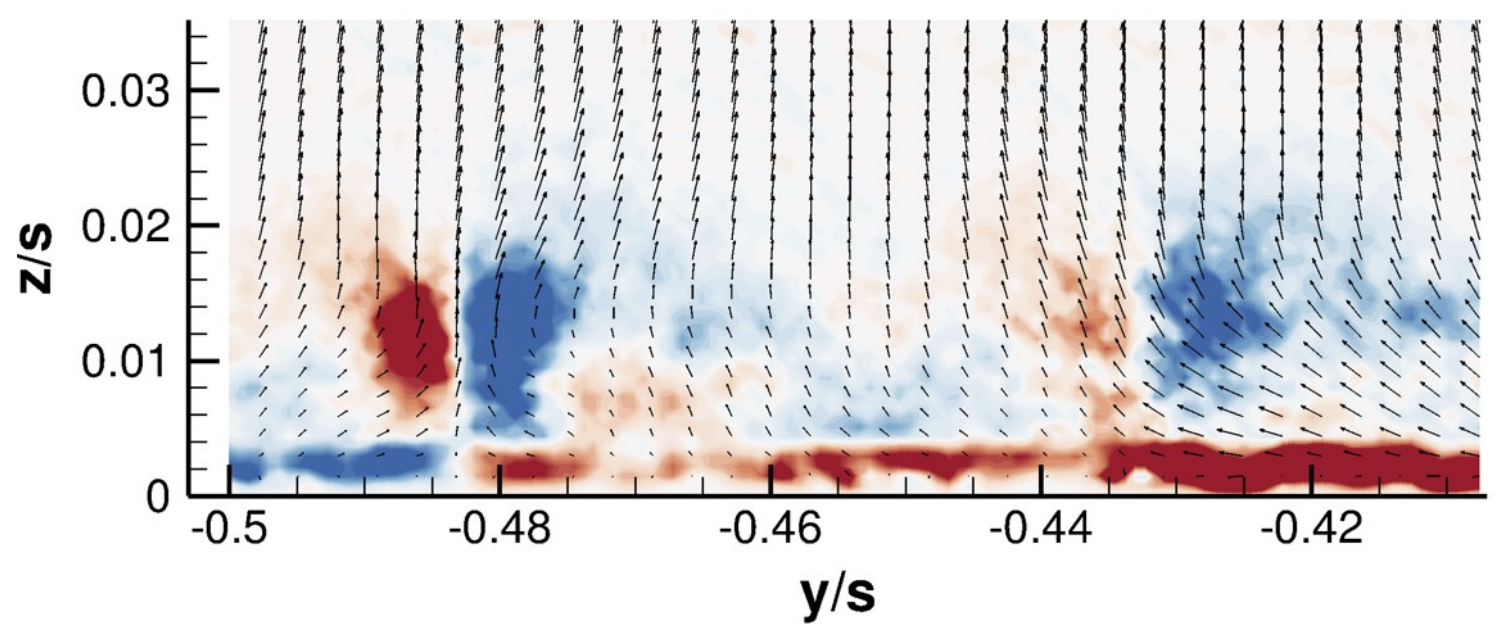

(b) Mod 2 Actuator, $P_{r}=1.7$.

Figure 11. Mean vorticity contours from spanwise PIV data. $R e_{c}=\mathbf{5 0 0 , 0 0 0 ,} \mathbf{M}=\mathbf{0 . 0 7}$, and $x / c=0.78$. 
flap of the NACA 0015 model. The flow visualization study suggests that the inferior performance of the original actuator can not be attributed to the SWJ actuators being spaced too far apart.

\section{Acknowledgments}

The authors would like to thank the Advanced Air Transport Technology Project of the NASA Advanced Air Vehicles Program for funding the research and the following individuals for their support; Catherine McGinley, Luther Jenkins, Judi Hannon, Norman Schaeffler, Donald Day, Christal Kellam, and Charlie Debro. Thanks are also extended to Mark Fletcher, Luther Jenkins, and Chung-sheng Yao for help with the PIV acquisition and analysis. The authors also thank model designer Sandy Webb for his work on the model spar modifications and SWJ actuator arrays. Thanks are extended to Wayne Geouge, Tom Hall, Kevin McLain, Robert Andrews, and Gary Wainwright for help with various aspects of the model fabrication and instrumentation process.

\section{References}

${ }^{1}$ McClean, J. D., Crouch, J. D., Stoner, R. C., Sakurai, S., Feifel, G. E., Feifel, W. M., and Rush, H. M., "Study of the Application of Separation Control by Unsteady Excitation to Civil Transport Aircraft," NASA/CR 1999-209338, 1999.

${ }^{2}$ Gregory, J. W. and Tomac, M., "A Review of Fluidic Oscillator Development and Application for Control," AIAA Paper 2013-2474, June 2013.

${ }^{3}$ Andino, M. Y., Lin, J. C., Washburn, A. E., Whalen, E. A., Graff, E. C., and Wygnanski, I. J., "Flow Separation Control on a Full-Scale Vertical Tail Model using Sweeping Jet Actuators," AIAA Paper 2015-0785, Jan. 2015.

${ }^{4}$ Whalen, E. A., Lacy, D. S., Lin, J. C., Andino, M. Y., Washburn, A. E., Graff, E. C., and Wygnanski, I. J., "Performance Enhancement of a Full-Scale Vertical Tail Model Equipped with Active Flow Control," AIAA Paper 2015-0784, Jan. 2015.

${ }^{5}$ Lin, J. C., Andino, M. Y., Alexander, M. G., Whalen, E. A., Spoor, M. A., Tran, J. T., and Wygnanski, I. J., "An Overview of Active Flow Control Enhanced Vertical Tail Technology Development," AIAA Paper 2016-0056, Jan. 2016.

${ }^{6}$ Martin, P. B., Overmeyer, A. D., Tanner, P. E., Wilson, J. S., and Jenkins, L. N., "Helicopter Fuselage Active Flow Control in the Presence of a Rotor," American Helicopter Society 70th Annual Forum, Paper 335, Montréal, Québec, Canada, 2014.

${ }^{7}$ Seifert, A., Stalnov, O., Sperber, D., Arwatz, G., Palei, V., David, S., Dayan, I., and Fono, I., "Large Trucks Drag Reduction Using Active Flow Control," AIAA Paper 2008-0743, Jan. 2008.

${ }^{8}$ Wilson, J., Schatzman, D., Arad, E., Seifert, A., and Shtendel, T., "Suction and Pulsed-Blowing Flow Control Applied to an Axisymmetric Body," AIAA Journal, Vol. 51, No. 10, 2013, pp. 2432-2446.

${ }^{9}$ Koklu, M., "The Effects of Sweeping Jet Actuator Parameters on Flow Separation Control," AIAA Paper 2015-2485, June 2015.

${ }^{10}$ Cerretelli, C., Wuerz, W., and Gharaibah, E., "Unsteady Separation Control on Wind Turbine Blades using Fluidic Oscillators," AIAA Journal, Vol. 48, No. 7, July 2010, pp. 1302-1311.

${ }^{11}$ Woszidlo, R. and Wygnanski, I., "Parameters Governing Separation Control with Sweeping Jet Actuators," AIAA Paper 2011-3172, June 2011.

${ }^{12}$ Woszidlo, R., Nawroth, H., Raghu, S., and Wygnanski, I., "Parametric Study of Sweeping Jet Actuators for Separation Control," AIAA Paper 2010-4247, July 2010.

${ }^{13}$ Pack Melton, L. G., "Active Flow Separation Control on a NACA 0015 Wing using Fluidic Actuators," AIAA Paper 2014-2364, June 2014.

${ }^{14}$ Vatsa, V., Koklu, M., and Wygnanski, I., "Numerical Simulation of Fluidic Actuators for Flow Control Applications," AIAA Paper 2012-3239, June 2012.

${ }^{15}$ Seele, R., Graff, E., Gharib, M., Taubert, L., Lin, J., and Wygnanski, I., "Improving Rudder Effectiveness with Sweeping Jet Actuators," AIAA Paper 2012-417, June 2012.

${ }^{16}$ Pack Melton, L. G. and Koklu, M., "Active Flow Control Using Sweeping Jet Actuators on a Semi-Span Wing Model," AIAA Paper 2016-1872, Jan. 2016.

${ }^{17}$ Seele, R., Graff, E., Gharib, M., Taubert, L., Lin, J., and Wygnanski, I., "Performance Enhancement of a Vertical Tail Model with Sweeping Jet Actuators," AIAA Paper 2013-0411, Jan. 2013.

${ }^{18}$ Hartwich, P. M., Shmilovich, A., Lacy, D. S., Dickey, E. D., Sclafani, A. J., Sundaram, P., and Yadlin, Y., "Refined AFC-Enabled High-Lift System Integration Study," NASA/CR 2016-219170, 2016.

${ }^{19}$ Milholen, W., Jones, G., and Chan, D., "High-Reynolds Number Circulation Control Testing in the National Transonic Facility (invited)," AIAA Paper 2012-0103, Jan. 2012.

${ }^{20}$ Milholen, W. E., Jones, G. S., Chan, D. T., Goodliff, S., Anders, S., Pack Melton, L. G., Carter, M. B., Allan, B. G., and Capone, F., "Enhancements to the FAST-MAC Circulation Control Model and Recent High-Reynolds Number Testing in the National Transonic Facility," AIAA Paper 2013-2794, June 2013.

${ }^{21}$ Greenblatt, D. and Washburn, A., "Influence of Finite Span and Sweep on Active Flow Control Efficacy," AIAA Journal, Vol. 46, No. 7, 2008, pp. 1675-1694.

${ }^{22}$ Greenblatt, D., "Dual Location Separation Control on a Semispan Wing," AIAA Journal, Vol. 45, No. 8, 2007, pp. 18481860. 
${ }^{23}$ Greenblatt, D., "Management of Vortices Trailing Flapped Wings via Separation Control," AIAA Paper 2005-0061, Jan. 2005.

${ }^{24}$ Koklu, M. and Melton, L., "Sweeping Jet Actuator in a Quiescent Environment," AIAA Paper 2013-2477, June 2013.

${ }^{25}$ Koklu, M. and Owens, L. R., "Flow Separation Control Over a Ramp Using Sweeping Jet Actuators," AIAA Paper 2014-2367, June 2014.

${ }^{26}$ Owens, L., Kegerise, M., and Wilkinson, S., "Off-Body Boundary-Layer Measurement Techniques Development for Supersonic Low-Disturbance Flows," AIAA Paper 2011-0284, June 2011. 